International Mathematical Forum, 2, 2007, no. 63, 3115 - 3123

\title{
On Quasi Quadratic Functionals and Existence of Related Sesquilinear Functionals
}

\author{
Mehmet Açıkgöz \\ University of Gaziantep, Faculty of Science and Arts \\ Department of Mathematics, 27310 Gaziantep, Turkey \\ acikgoz@gantep.edu.tr \\ Aliakbar Goshabulaghi \\ Faculty of Mathematics and Computer Sciences \\ Amirkabir University of Technology (Tehran Polytechnic) \\ 424 Hafez Ave., Tehran 15914, Iran \\ alakgoo@yahoo.com
}

\begin{abstract}
In this study, a general solution of the problem of the characterization of quasi-quadratic functionals posed by P. Śemrl is given. Then we will generalize this characterization twice which includes quasi-quadratic functional on quaternion spaces.
\end{abstract}

Mathematics Subject Classification: Primary 11E04, 46C05, Secondary 11D09

Keywords: Quadratic functional, quasi-quadratic functional, sesquilinear functional, quaternion space, Jordan *-derivation

\section{Introduction ANd Preliminaries}

Let $M$ be a module over a *-ring $R$. A mapping $S: M \times M \rightarrow R$ is called a sesquilinear functional if it is linear in the first argument and antilinear in the second argument:

$$
\begin{gathered}
S(a x+b y, z)=a S(x, z)+b S(y, z), \quad x, y, z \in M, \quad a, b \in R \\
S(x, a y+b z)=S(x, y) a^{*}+S(x, z) b^{*}, \quad x, y, z \in M, \quad a, b \in R
\end{gathered}
$$

A quadratic functional $Q$ on $M$ is defined as the composition of some sesquilinear functional from $M \times M$ to $R$ with the diagonal injection of $M$ into $M \times M$; that is, $Q(x)=S(x, x)$, where $S$ is sesquilinear. Now there is a question of what requirements can be imposed on a mapping from $M$ to $R$ to 
define the set of all quadratic functionals. The best-known identities satisfied by quadratic functionals are the parallelogram law

$$
Q(x+y)+Q(x-y)=2 Q(x)+2 Q(y), \quad x, y \in M,
$$

and the homogeneity equation

$$
Q(a x)=a Q(x) a^{*}, \quad x \in M, a \in R .
$$

A mapping $Q: M \rightarrow R$ satisfying these two identities is called a quasiquadratic functional.

It seems natural to ask when quasi quadratic functionals are quadratic functionals. In 1963, Halperin in his lecture on Hilbert spaces posed this problem for the special case that $M$ is a vector space over $F \in\{R, C, H\}$. Here, $R$ and $C$ denote the field of real and complex numbers respectively and $H$ denotes the skew-field of quaternions. In 1987 Šemrl [3] gave a positive answer to Halperin's problem for quasi-quadratic functionals defined on a vector space over a complex *-algebra with an identity. It was proved that if $Q$ is a quasiquadratic functional on a module over a complex *-algebra with an identity element, then the mapping $S$ defined by

$$
S(x, y)=\frac{1}{4}(Q(x+y)-Q(x-y))+\frac{i}{4}(Q(x+i y)-Q(x-i y))
$$

is sesquilinear functional and satisfies $Q(x)=S(x, x)$. This result is an extension of the Jordan-Von Neumann theorem [4] which characterises pre-Hilbert spaces among all normed spaces. Here we will extend this result to moduls on *-rings having an element or elements with special properties including complex or quaternionic spaces [1] which will introduce later.

A mapping $J$ defined on a ${ }^{*}$-ring $R$ is called a Jordan ${ }^{*}$-derivation if it is additive and satisfies $J\left(a^{2}\right)=a J(a)+J(a) a^{*}$. A mapping $J_{a}: R \rightarrow R, a \in R$, defined by $J_{a}(b)=b a-a b^{*}$ will be called an inner Jordan *-derivation [4].

\section{Main Results}

Theorem 1. Let $M$ be a left module on the ${ }^{*}$-ring $R$ with identity such that 2 be a unit in $R$ and an element $a_{0} \in R$ exists, such that

$$
\begin{aligned}
& a_{0}+a_{0}^{*}=0, \quad a_{0} a_{0}^{*}=1 \\
& a_{0} a=a a_{0}, \quad(a \in R)
\end{aligned}
$$

also let $Q: M \rightarrow R$ be a quasi-quadratic functional. Then the mapping $S$ : $M \times M \rightarrow R$ defined by

$$
S(x, y)=\frac{1}{4}(Q(x+y)-Q(x-y))+\frac{a_{0}}{4}\left(Q\left(x+a_{0} y\right)-Q\left(x-a_{0} y\right)\right)
$$

is sesquilinear and $Q(x)=S(x, x)$ holds for any $x \in M$. 
Proof. Since

$$
\begin{aligned}
Q\left(x+a_{0} x\right)-Q\left(x-a_{0} x\right)= & \left(1+a_{0}\right) Q(x)\left(1+a_{0}^{*}\right) \\
& -\left(1-a_{0}\right) Q(x)\left(1-a_{0}^{*}\right) \\
= & 2\left(a_{0} Q(x)+Q(x) a_{0}^{*}\right)=0,
\end{aligned}
$$

So we have $S(x, x)=\frac{1}{4} Q(2 x)=Q(x)$. Now let

$S_{1}(x, y)=Q(x+y)-Q(x-y), \quad S_{2}(x, y)=a_{0}\left(Q\left(x+a_{0} y\right)-Q\left(x-a_{0} y\right)\right)$,

then from (3) we have

$$
\begin{aligned}
2 S_{1}\left(x_{1}, y\right)+2 S_{1}\left(x_{2}, y\right)= & 2 Q\left(x_{1}+y\right)-2 Q\left(x_{1}-y\right) \\
& +2 Q\left(x_{2}+y\right)-2 Q\left(x_{2}-y\right) \\
= & Q\left(x_{1}+x_{2}+2 y\right)+Q\left(x_{1}-x_{2}\right) \\
& -Q\left(x_{1}+x_{2}-2 y\right)-Q\left(x_{1}-x_{2}\right) \\
= & Q\left(x_{1}+x_{2}+2 y\right)-Q\left(x_{1}+x_{2}-2 y\right) \\
= & S_{1}\left(x_{1}+x_{2}, 2 y\right) .
\end{aligned}
$$

Similarly we have $2 S_{1}\left(x, y_{1}\right)+2 S_{1}\left(x, y_{2}\right)=S_{1}\left(2 x, y_{1}+y_{2}\right)$. Therefore we can write

$$
2 S_{2}\left(x_{1}, y\right)+2 S_{2}\left(x_{2}, y\right)=a_{0} S_{1}\left(x_{1}+x_{2}, 2 a_{0} y\right)=S_{2}\left(x_{1}+x_{2}, 2 y\right),
$$

and

$$
2 S_{2}\left(x, y_{1}\right)+2 S_{2}\left(x, y_{2}\right)=a_{0} S_{1}\left(2 x, a_{0}\left(y_{1}+y_{2}\right)\right)=S_{2}\left(2 x, y_{1}+y_{2}\right)
$$

Hence

$$
\begin{aligned}
2 S\left(x_{1}, y\right)+2 S\left(x_{2}, y\right) & =\frac{1}{4}\left(2 S_{1}\left(x_{1}+x_{2}, 2 y\right)+2 S_{2}\left(x_{1}+x_{2}, 2 y\right)\right) \\
& =S\left(x_{1}+x_{2}, 2 y\right)
\end{aligned}
$$

Now by giving $x_{2}=0$ in $(2.3)$ we get $S\left(x_{1}, y\right)=\frac{1}{2} S\left(x_{1}, 2 y\right)$. Hence $S\left(x_{1}, y\right)+$ $S\left(x_{2}, y\right)=S\left(x_{1}+x_{2}, y\right)$. Similarly the identity $S\left(x, y_{1}\right)+S\left(x, y_{2}\right)=S\left(x, y_{1}+y_{2}\right)$ 
holds and therefore $S$ is biadditive. By using (1.4) we have

$$
\begin{aligned}
S\left(a_{0} x, y\right)= & \frac{1}{4}\left(Q\left(a_{0} x-a_{0}^{2} y\right)-Q\left(a_{0} x+a_{0}^{2} y\right)\right) \\
& +\frac{a_{0}}{4}\left(Q\left(a_{0} x+a_{0} y\right)-Q\left(a_{0} x-a_{0} y\right)\right) \\
= & \frac{1}{4}\left(Q\left(x-a_{0} y\right)-Q\left(x+a_{0} y\right)\right) \\
& +\frac{a_{0}}{4}(Q(x+y)-Q(x-y)) \\
= & a_{0}\left[\frac{1}{4}(Q(x+y)-Q(x-y))\right. \\
& \left.+\frac{a_{0}}{4}\left(Q\left(x+a_{0} y\right)-Q\left(x-a_{0} y\right)\right)\right] \\
= & a_{0} S(x, y)=S(x, y) a_{0},
\end{aligned}
$$

Similarly the identity $S\left(x, a_{0} y\right)=S(x, y) a_{0}^{*}=a_{0}^{*} S(x, y)$ holds and since $S(c x, c y)=c S(x, y) c^{*}$ hence we have

$$
\begin{aligned}
& S(a b x, a y)+S(a b x, y)+S(b x, a y)+S(b x, y) \\
= & S((a+1) b x,(a+1) y) \\
= & (a+1) S(b x, y)\left(a^{*}+1\right) \\
= & a S(b x, y) a^{*}+a S(b x, y)+S(b x, y) a^{*}+S(b x, y),
\end{aligned}
$$

So the identity

$$
S(a b x, y)+S(b x, a y)=a S(b x, y)+S(b x, y) a^{*},
$$

holds and replacing $a$ with $a_{0} a$ we conclude

$$
S(a b x, y)-S(b x, a y)=a S(b x, y)-S(b x, y) a^{*} .
$$

Comparing (2.5) and (2.6) we get

$$
S(a b x, y)=a S(b x, y), \quad S(b x, a y)=S(b x, y) a^{*} .
$$

Hence $S$ is sesquilinear.

Notice 1. By putting $a_{0}=i .1$ in complex *algebras, this theorem yields Šmrl's theorem [3]. Note that for complex *-algebras, $a_{0}=i .1$ has obtained properties.

If there exists an element $a_{0} \in R$ with properties (2.1), and (2.2) then for any element $r \in R$ by giving $r_{1}=\frac{1}{2}\left(r+r^{*}\right)$ and $r_{1}=\frac{a_{0}^{*}}{2}\left(r-r^{*}\right)$ we get the representation $r=r_{1}+a_{0} r_{2}$. Moreover this representation is the unique one such that $r_{1}^{*}=r_{1}, r_{2}^{*}=r_{2}$.

Proposition 1. Let $M$ be a left module on the ${ }^{*}{ }_{-}$ring $R$ with identity such that 2 be $a$ unit in $R$ and there exist an element $a_{0} \in R$ satisfyinf (2.1) and (2.2). 
If $S: M \times M \rightarrow R$ be a sesquilinear functional, then there exist sesquilinear functionals $S_{1}, S_{2}: M \times M \rightarrow R$ such that

$$
\begin{gathered}
S(x, y)=S_{1}(x, y)+a_{0} S_{2}(x, y), \\
S_{1}(x, y)^{*}=S_{1}(y, x), \quad S_{2}(x, y)^{*}=S_{2}(y, x) .
\end{gathered}
$$

Moreover $S_{1}$ and $S_{2}$ are unique sesquilinear functionals satisfying (2.7), and $(2.8)$.

Proof. Let

$$
S_{1}(x, y)=\frac{1}{2}\left(S(x, y)+S(y, x)^{*}\right), \quad S_{2}(x, y)=\frac{a_{0}^{*}}{2}\left(S(x, y)-S(y, x)^{*}\right) .
$$

Theorem 2. Let $R$ be $a^{*}{ }^{*}$-ring with identity 1 such that 2 is a unit in $R$. Moreover,

(1) There exists a finite subset $A$ of $R$ such that any $a_{k} \in A$ satisfies (2.1).

(2) $G=\{-1,1\} \cup A$ is a group relative to the product of $R$.

(3) For any $r \in R, a_{k} r=r a_{k}$ or $a_{k} r=-r a_{k}$.

(4) If for some $r \in R$, the equality $a_{k} r a_{k}=r$ for any $a_{k} \in G$ holds then $r=0$.

(5) The mapping $Q: M \rightarrow R$ be a quasi-quadratic functional such that $a_{k} r=r a_{k},\left(a_{k} \in G, r \in \operatorname{Im} Q\right)$

Then the functional $S: M \times M \rightarrow R$ defined by

$$
S(x, y)=\frac{1}{4} \sum_{a_{k} \in G} a_{k} Q\left(x+a_{k} y\right)
$$

is sesquilinear and $S(x, y)=Q(x)$. 
Proof. $S$ is bilinear since the functional $S_{1}: M \times M \rightarrow R$ defined by $S_{1}(x, y)=$ $Q(x+y)-Q(x-y)$ is bilinear. Also

$$
\begin{aligned}
S(x, x) & =\frac{1}{4} \sum_{a_{k} \in G} a_{k} Q\left(x+a_{k} x\right)=\frac{1}{4} \sum_{a_{k} \in G} a_{k} Q\left(\left(1+a_{k}\right) x\right) \\
& =\frac{1}{4} \sum_{a_{k} \in G} a_{k}\left(1+a_{k}\right) Q(x)\left(1+a_{k}^{*}\right) \\
& =Q(x)+\frac{1}{4} \sum_{a_{k} \in G \backslash\{ \pm 1\}}\left(a_{k}-1\right) Q(x)\left(1+a_{k}^{*}\right) \\
& =Q(x)+\frac{1}{4} \sum_{a_{k} \in G \backslash\{ \pm 1\}}\left[a_{k} Q(x) a_{k}^{*}-Q(x)+a_{k} Q(x)-Q(x) a_{k}^{*}\right] \\
& =Q(x)+\frac{1}{4} \sum_{a_{k} \in G \backslash\{ \pm 1\}} a_{k} Q(x)+\frac{1}{4} \sum_{a_{k} \in G \backslash\{ \pm 1\}} Q(x) a_{k}^{*} \\
& =Q(x)
\end{aligned}
$$

Now let $c \in R$, if $a_{k} c=c a_{k}$ then $a_{k}^{*} c=c a_{k}^{*}$ and $a_{k} Q\left(c x+a_{k} c y\right)=$ $c\left(a_{k} Q\left(x+a_{k} y\right)\right) c^{*}$, and if $a_{k} c=-c a_{k}$ then $a_{k}^{*} c=-c a_{k}^{*}$ and $a_{k} Q\left(c x+a_{k} c y\right)=$ $a_{k} Q\left(c x-c a_{k} y\right)=c\left(-a_{k} Q\left(x-a_{k} y\right)\right) c^{*}$. Thus

$$
\begin{aligned}
S(c x, c y) & =\frac{1}{4} \sum_{a_{k} \in G} a_{k} Q\left(c x+a_{k} c y\right) \\
& =\frac{1}{4} \sum_{a_{k} \in G_{1}} c a_{k} Q\left(x+a_{k} y\right) c^{*}+\frac{1}{4} \sum_{a_{k} \in G_{2}} c\left(-a_{k} Q\left(x-a_{k} y\right)\right) c^{*} \\
& =c\left(\frac{1}{4} \sum_{a_{k} \in G} a_{k} Q\left(x+a_{k} y\right)\right) c^{*} \\
& =c S(x, y) c^{*}
\end{aligned}
$$

Since $G_{1}$ and $G_{2}$ are closed to the involution. Hence similar to theorem 1, we have

$$
S(a b x, y)+S(b x, a y)=S(b x, y) a^{*}+a S(b x, y)
$$

Also we have

$$
\begin{aligned}
S\left(a_{j} x, y\right) & =\frac{1}{4} \sum_{a_{k} \in G} a_{k} Q\left(a_{j} x+a_{k} y\right)=\frac{1}{4} \sum_{a_{k} \in G} a_{j} a_{j}^{*} a_{k} Q\left(x+a_{j}^{*} a_{k} y\right) \\
& =a_{j} S(x, y), \quad\left(a_{j} \in G\right)
\end{aligned}
$$

Similarly for $a_{j} \in G$ the equality $S\left(x, a_{j} y\right)=S(x, y) a_{j}^{*}$ holds. Now by replacing $a$ with $a_{j} a$ in $(2.9)$ we get

$$
a_{j} S(a b x, y)+S(b x, a y) a_{j}^{*}=S(b x, y) a^{*} a_{j}^{*}+a_{j} a S(b x, y)
$$


This with (2.9) yields

$$
S(b x, a y)-S(b x, y) a^{*}=a_{j}\left(S(b x, a y)-S(b x, y) a^{*}\right) a_{j}
$$

Hence by the hypothesis of theorem $S(b x, a y)=S(b x, y) a^{*}$ and as a result $S(b x, a y)=b S(x, y) a^{*}$ holds.

Theorem 3. Let $R$ be $a^{*}$-ring with identity 1 such that 2 is a unit in $R$. Moreover,

(1) There exists a finite subset $A$ of $R$ such that any $a_{k} \in A$ satisfies (2.1).

(2) $G=\{-1,1\} \cup A$ is a group relative to the product of $R$.

(3) For any $r \in\left\{r: r=r^{*}\right\}$ and $a_{k} \in G, a_{k} r=r a_{k}$.

(4) For any $c \in R,\left\{c_{i}\right\} \subset\left\{r: r=r^{*}\right\}$ exists such that $c=\sum_{i} c_{i} a_{i}$.

(5) If for some $r \in R$, the equality $a_{k} r a_{k}=r$ for any $a_{k} \in G$ holds then $r=0$.

(6) The mapping $Q: M \rightarrow R$ be a quasi-quadratic functional such that $a_{k} r=r a_{k},\left(a_{k} \in G, r \in \operatorname{Im} Q\right)$.

Then the mapping $S . M \times M \rightarrow R$ defined by

$$
S(x, y)=\frac{1}{4} \sum_{a_{k} \in G} a_{k} Q\left(x+a_{k} y\right)
$$

is sesquilinear and $S(x, x)=Q(x)$.

Proof. Similar to theorem $2, S$ is bilinear. It is clear that for any $c \in R$ which $c=c^{*}$ the property $S(c x, c y)=c S(x, y) c$ satisfies. Also

$$
\begin{aligned}
S\left(a_{i} x, y\right) & =\frac{1}{4} \sum_{a_{k} \in G} a_{k} Q\left(a_{i} x+a_{k} y\right)=\frac{1}{4} \sum_{a_{k} \in G} a_{i} a_{i}^{*} a_{k} Q\left(x+a_{i}^{*} a_{k} y\right) \\
& =a_{i} S(x, y),
\end{aligned}
$$

and

$$
\begin{aligned}
S\left(x, a_{j} y\right) & =\frac{1}{4} \sum_{a_{k} \in G} a_{k} Q\left(x+a_{k} a_{j} y\right)=\frac{1}{4} \sum_{a_{k} \in G} a_{k} a_{j} a_{j}^{*} Q\left(x+a_{k} a_{j} y\right) \\
& =S(x, y) a_{j}^{*} .
\end{aligned}
$$

So for any $c \in R$ we have

$$
\begin{aligned}
S(c x, c y) & =S\left(\sum_{i} c_{i} a_{i} x, \sum_{j} c_{j} a_{j} y\right)=\sum_{i} \sum_{j} S\left(c_{i} a_{i} x, c_{j} a_{j} y\right) \\
& =\sum_{i} \sum_{j} c_{i} a_{i} S(x, y)\left(c_{j} a_{j}\right)^{*}=\left(\sum_{i} c a_{i}\right) S(x, y)\left(\sum_{j} c_{j} a_{j}\right)^{*} \\
& =c S(x, y) c^{*} .
\end{aligned}
$$

Now one can prove the remainder of theorem similar to theorem 2. 
Notice 2. Any quaternion space $H$ posses the conditions of theorem 3 but $H$ does not posse condition (3) of theorem 2. Note that both theorems are generalizing theorem 1 .

Theorem 4. Let $R$ be $a^{*}$-ring with identity 1 such that 2 is not a zerodivisor. If $J: R \rightarrow R$ is a Jordan ${ }^{*}$-derivation then the mapping $Q: R \times R \rightarrow R$ given by $Q(a, b)=J(b a)-b J(a)-J(a) b^{*}$ is a quasi-quadratic functional. If $J$ is not inner then $Q$ is not a quadratic functional.

Proof. [[2], theorem 2].

Now consider two different cases on $R$. First, let there exist $b_{0} \in R$ such that $b_{0} \neq b_{0}^{*}$, then by considering $a_{0}=\frac{1}{2}\left(b_{0}-b_{0}^{*}\right)$ we get $a_{0}+a_{0}^{*}=0$. In this case we want to know if any quasi-quadratic functional on $M$ be quadratic, does one of such elements satisfies the equation $a_{0} a_{0}^{*}=1$ ? From theorem 4 we know that for any Jordan ${ }^{*}$-derivation specially $J_{a_{0}}$ the mapping

$$
Q(a, b)=J_{a_{0}}(b a)-b J_{a_{0}}(a)-J_{a_{0}}(a) b^{*}=b a_{0} a^{*}-a a_{0} b^{*}
$$

is quadratic ( moreover from theorem 4 any Jordan *-derivation on $R$ is inner if any quasi-quadratic functional be quadratic). If we could show for some $a_{0}$ introduced above the equation $2 a_{0} a_{0}^{*}=Q\left(a_{0}, 1\right)=2$ holds then we almost get the inverse case of theorem 1 . This question will remain open here. Second, if for any $a \in R, a=a^{*}$, then the following theorem is proven which shows that the inverse case of recent theorems (existing an element satisfying identity (2.1) ) may be not true in general.

Theorem 5. Let $R$ be a commutative *-ring having identity and 2 as a unit in $R$ with trivial involution on a module $M$ such that each Jordan *-derivation on $R$ be inner and hence zero. Then for any quasi quadratic functional $Q$ : $M \rightarrow R$, the mapping $S: M \times M \rightarrow R$ defined by

$$
S(x, y)=\frac{1}{4}(Q(x+y)-Q(x-y))
$$

is sesquilinear and for any $x \in M$, we have $S(x, x)=Q(x)$.

Proof. For any trivial fixed $x, y \in M$ define the mapping $D: R \rightarrow R$ by

$$
D(a)=S(a x, y)-a S(x, y) .
$$


We show that $D$ is Jordan *-derivation and hence $D=0$. $D$ is additive since $S$ is biadditive. Moreover

$$
\begin{aligned}
4 S\left(a^{2} x, y\right)+4 a^{2} S(x, y)= & Q\left(a^{2} x+y\right)-Q\left(a^{2} x-y\right) \\
& +a^{2} Q(x+y)-a^{2} Q(x-y) \\
= & Q\left(a^{2} x+y\right)-Q\left(a^{2} x-y\right) \\
& +Q(a x+a y)-Q(a x-a y) \\
= & \frac{1}{2}\left(Q\left(\left(a^{2}+a\right) x+(a+1) y\right)\right. \\
& +Q\left(\left(a^{2}-a\right) x+(1-a) y\right) \\
& -Q\left(\left(a^{2}+a\right) x-(1+a) y\right) \\
& \left.-Q\left(\left(a^{2}-a\right) x+(a-1) y\right)\right) \\
= & \frac{1}{2}\left[(a+1)^{2} Q(a x+y)+(a-1)^{2} Q(a x-y)\right. \\
& \left.-(a+1)^{2} Q(a x-y)-(a-1)^{2} Q(a x+y)\right] \\
= & 2 a Q(a x+y)-2 a Q(a x-y) \\
= & 8 a S(a x, y) .
\end{aligned}
$$

Thus

$$
D\left(a^{2}\right)=S\left(a^{2} x, y\right)-a^{2} S(x, y)=2 a S(a x, y)-2 a^{2} S(x, y)=2 a D(a) .
$$

\section{REFERENCES}

[1] Acikgoz, M., Emirhan, B., On the representation of quasi-quadratic functional by sesquilinear ones over real $(R)$, complex $(C)$ and quaternionic $(H)$ vector spaces, Advanced Studies in Cont. Math., 11, no 1 (2005), 85-96.

[2] Šemrl, P., Quadratic and quasi-quadratic functionals, Proc. Amer. Math. Soc., 119, no 4 (1993), 1105-1113.

[3] Šemrl, P., On quadratic functionals, Bull. Austral. Math. Soc., 37 (1987), 27-28.

[4] Jordan, P., Von Neumann, J., On inner products in linear metric spaces, Ann. of Math.(2), 36 (1935), 719-723.

\section{Received: June 23, 2007}

